\title{
Arctic soil patterns analogous to fluid instabilities
}

\author{
Rachel C. Glade ${ }^{\mathrm{a}}$, Michael Fratkin ${ }^{\mathrm{a}}$, Mehdi Pouragha ${ }^{\mathrm{b}}$, \\ Ali Seiphooric , and Joel C. Rowland ${ }^{a}$
}

May 23, 2021

a) EES-14, Los Alamos National Lab, Los Alamos, NM, 87545. b) Department of Civil and Environmental Engineering, Carleton University, 1125 Colonel By Drive, Ottawa, ON, K1S

5B6. c) Department of Earth, Atmospheric and Planetary Sciences, Massachusetts Institute of Technology, Green Bldg, The, 77 Massachusetts Ave, Cambridge, MA 02139

This manuscript has now been published at PNAS:

https://www.pnas.org/content/118/21/e2101255118/tab-article-info. If you would like to cite this work, please refer to the final published version and cite:

Glade, Rachel C. and Fratkin, Michael M. and Pouragha, Mehdi and Seiphoori, Ali and Rowland, Joel C. Arctic soil patterns analogous to fluid instabilities, Proceedings of the National Academy of Sciences, vol. 118, 21. 10.1073/pnas.2101255118 


\title{
Arctic soil patterns analogous to fluid instabilities
}

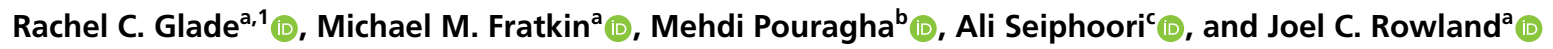 \\ ${ }^{a}$ Earth and Environmental Sciences Division, Los Alamos National Laboratory, Los Alamos, NM 87545; ${ }^{b}$ Department of Civil and Environmental Engineering, \\ Carleton University, Ottawa, ON K1S 5B6, Canada; and 'Department of Earth, Atmospheric and Planetary Sciences, Massachusetts Institute of Technology, \\ Cambridge, MA 02139
}

Edited by Michael Manga, University of California, Berkeley, CA, and approved April 19, 2021 (received for review January 22, 2021)

\begin{abstract}
Slow-moving arctic soils commonly organize into striking largescale spatial patterns called solifluction terraces and lobes. Although these features impact hillslope stability, carbon storage and release, and landscape response to climate change, no mechanistic explanation exists for their formation. Everyday fluids-such as paint dripping down walls-produce markedly similar fingering patterns resulting from competition between viscous and cohesive forces. Here we use a scaling analysis to show that soil cohesion and hydrostatic effects can lead to similar largescale patterns in arctic soils. A large dataset of high-resolution solifluction lobe spacing and morphology across Norway supports theoretical predictions and indicates a newly observed climatic control on solifluction dynamics and patterns. Our findings provide a quantitative explanation of a common pattern on Earth and other planets, illuminating the importance of cohesive forces in landscape dynamics. These patterns operate at length and time scales previously unrecognized, with implications toward understanding fluid-solid dynamics in particulate systems with complex rheology.
\end{abstract}

solifluction | fluid instabilities | climate | granular fingering | periglacial

$\mathbf{P}$ eriodically frozen soil-a temporally evolving mixture of granular material, fluid, and ice-is one of the most complex natural materials found on planetary surfaces. While its rheology is not well understood, arctic soil deformation commonly produces large, distinctive meters to tens of meters-scale spatial patterns visible in aerial images (Fig. $1 A$ and $B$ ). Patterns are organized in both the downslope and cross-slope directions. Regular downslope-oriented terraces of soil are characterized by raised fronts that protrude 1 to $2 \mathrm{~m}$ above the surrounding topography (Fig. $1 A$ and $C$ ). Terrace fronts are commonly broken into finger-like lobes evenly spaced cross-slope (Fig. $1 A$ and $B$ ). Known as solifluction features, these patterns form due to a combination of frost heave, in which segregation ice growth lofts soil upward, and gelifluction, a slow flow-like relaxation of partially saturated soil once it thaws in the summer $(1,2)$. While a rich history of experimental and global field observations over the past century has characterized solifluction processes and velocities $(\sim$ $10^{-1}$ to $\left.10^{1} \mathrm{~cm} / \mathrm{y}\right)(2,3)$, there exists no agreed-upon rheological model for solifluction that can offer quantitative and qualitative explanations for the striking patterns it produces. Renewed interest in these features primarily stems from a need to predict arctic landscape response to climate change and storage and release of permafrost carbon, as well as to predict and mitigate arctic slope instabilities due to thawing permafrost (4).

Strikingly similar patterns develop in simple fluids, where competition between viscous and cohesive forces drives a suite of common instabilities in thin films. For example, the evenly spaced fluid fingers that form when painting a wall, icing a cake, or sloshing oil in a frying pan are known as "contact line instabilities" at fluid fronts $(7,11)$ (Fig. $1 A$ ). Only recently have soft solids (12) and granular materials (13-18) been shown to exhibit patterns and morphology that resemble those of thin-film fluids. Notably, ref. 13 found that small cohesive forces between sand grains produce an effective surface tension relevant at macroscopic length scales, causing a steady stream of sand to break into droplets similar to a Rayleigh-Plateau instability. However, connections between fluid and granular instabilities-especially regarding the role of cohesion-remain a frontier in materials science.

Here we take a step toward utilizing quantitative connections with fluid and granular mechanics to better understand solifluction processes and patterns. We present a conceptual model of solifluction pattern formation in which solifluction lobes (resembling fluid fingers) arise as a cross-slope instability on the fronts of terraces (resembling waves) formed during an initial downslope instability (Fig. $1 A$ and $B$ ). While we present data for both instabilities, we focus mainly on the cross-slope patterns. First, we discuss how key ingredients that control fluid contact line instabilities - viscosity, velocity, fluid thickness, and surface tension-may translate to soil. By adopting an analogy between fluid and soil dynamics, we suggest a formal scaling analysis relating solifluction wavelengths to active soil thickness, topographic slope, and cohesion-driven effects at the soil front. Using highresolution topographic data from over 3,000 solifluction lobes across 25 sites in Norway, we show that scaling between solifluction wavelengths and slope, lobe thickness, and lobe front angle generally agrees with our theoretical analysis. Data from these sites show that lobe morphology is strongly correlated with elevation, which likely represents a climate control on solifluction processes due to the dependence of frost heave on mean annual temperature amplitude $\left(T_{a}\right)$ and mean annual air temperature (MAAT). We discuss how cohesion not only slows down soil motion but also results in a state change in soil behavior, with implications for Arctic landscape response to climate change and interpretation of past climates on Earth and other planets. Our work shows that even in creeping granular-fluid-ice materials,

\section{Significance}

Slow-moving arctic soils form patterns resembling those found in common fluids, such as paint and cake icing drips. Inspired by fluid instabilities, we develop a conceptual model for soil patterns and use mathematical analysis to predict their wavelength. In particular, we propose that soil patterns arise due to competition between gravity and cohesion or the "stickiness" of soil grains. We compare our theoretical predictions with a dataset of soil features from Norway, finding that soil patterns are controlled by fluid-like properties as well as climate. Our work provides a physical explanation for a common pattern on both Earth and Mars, with implications for our understanding of landscapes and complex materials composed of both granular and fluid components.

Author contributions: R.C.G. designed research; R.C.G. and M.M.F. performed research R.C.G., M.M.F., M.P., A.S., and J.C.R. analyzed data; and R.C.G., M.P., A.S., and J.C.R. wrote the paper.

The authors declare no competing interest.

This article is a PNAS Direct Submission.

This open access article is distributed under Creative Commons Attribution-NonCommercialNoDerivatives License 4.0 (CC BY-NC-ND).

${ }^{1}$ To whom correspondence may be addressed. Email: rachel.glade@rochester.edu.

This article contains supporting information online at https://www.pnas.org/lookup/suppl/ doi:10.1073/pnas.2101255118/-/DCSupplemental. 


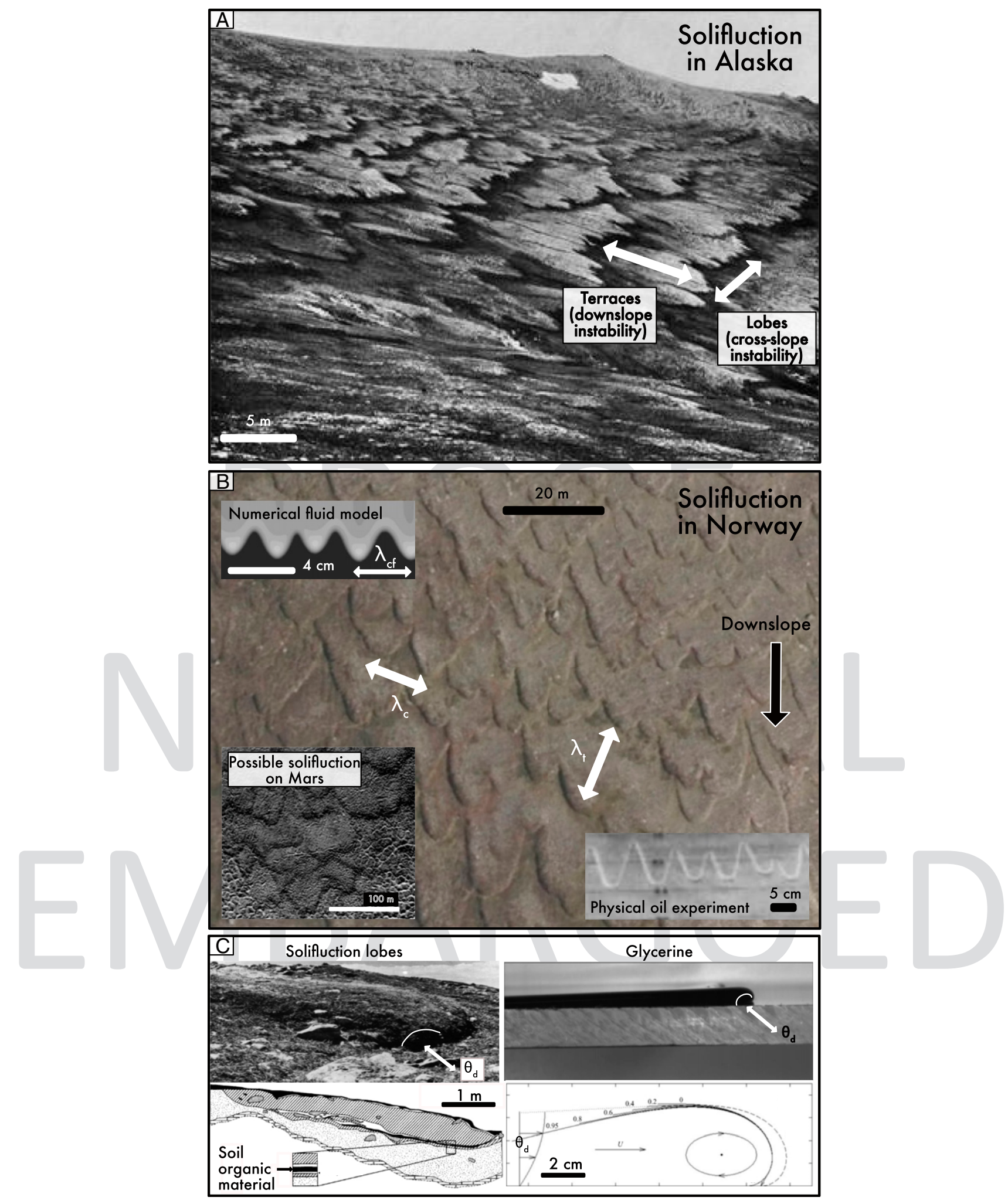

Fig. 1. (A) Solifluction terraces and lobes in Chicken Creek, AK. Photo by Philip S. Smith. Image credit: US Geological Survey. (B) Examples of solifluction and fluid patterns. Background: Orthophoto of solifluction lobes in Norway. Image credit: The Norwegian Mapping Authority. (Upper Left) Numerical model image of fluid fingering on an incline reprinted from ref. 5, with the permission of AIP Publishing. (Lower Left) Possible solifluction on Mars. Reprinted from ref. 6, with permission from Elsevier. (Lower Right) Photo of front of oil flowing down plane. Reprinted by permission of ref. 7: Springer Nature, Nature, copyright 1982. Cross-slope wavelengths for fluids $\lambda_{c f}$ and solifluction lobes $\lambda_{c}$ are shown, as well as the downslope solifluction terrace wavelength $\lambda_{t}$. (C) Morphology and dynamics of solifluction lobes vs. surface tension-dominated flows. (Upper Left) Solifluction lobe in Colorado. Reprinted from ref. 8, copyright Taylor and Francis. (Lower Left) Map of trenched lobe in Norway, with soil organic layer showing rollover motion. Adapted with permission from ref. 9. (Upper Right) Gravity-driven glycerine front. Reprinted with permission from ref. 10. (Lower Right) Schematic of glycerine front showing rollover motion. Reprinted with permission from ref. 10. Shape of nose is derived from Young-Laplace equation for surface tension effects. Numbers indicate profile evolution through time, and dashed line illustrates profile at next moment in time. Dynamic contact angle $\theta_{d}$ is shown for both a solifluction lobe and a fluid finger. 
competition between driving stress and cohesion can result in large-scale patterns similar to those found in fluids, with implications for our understanding of the rheological behavior of complex materials.

\section{Fluid Fingering Instabilities}

First, we briefly describe fingering instabilities in fluid films. The qualitative explanation for contact line instabilities is simple: At a fluid interface in a thin film, cohesive forces in the form of surface tension hold back the flow, allowing the front to thicken into a capillary ridge. With a slight initial perturbation, competition between body forces, which cause thicker zones to move faster, and surface tension, which induces transverse flow under bumps, drives the growth of fingers with a regular wavelength. Experiments (e.g., refs. 7, 19, and 20), linear stability analysis (e.g., refs. 11 and 21), and numerical models (e.g., refs. 5 and 22) have determined that the cross-slope wavelength $\lambda_{c f}$ of fluid contact line instabilities scales as

$$
\lambda_{c f} \sim h\left(\frac{\sigma}{3 v \mu}\right)^{1 / 3}
$$

where $h$ is the fluid thickness, $\mu$ is the fluid dynamic viscosity, $v$ is a characteristic velocity, $\sigma$ is the surface tension, and $\sigma / v \mu$ is the inverse capillary number $\mathrm{Ca}$. This means that flows with greater thickness or surface tension produce larger wavelengths, while more viscous or faster moving flows produce smaller wavelengths. While absolute finger wavelengths may differ depending on rheology, contact line instabilities have been shown to exhibit the scaling shown in Eq. 1 regardless of rheology $(19,22)$.
Note that $v$ depends on both $\mu$ and $h$; therefore, for a laminar Newtonian fluid with density $\rho$ flowing down a plane with slope angle $\phi$, in which we use a characteristic average velocity $v=\rho g h^{2} \sin \phi / 2 \mu$, Eq. 1 becomes

$$
\lambda_{c f} \sim\left(\frac{2 h \sigma}{3 \rho g \sin \phi}\right)^{1 / 3} .
$$

$\mathrm{Ca}$ has also been shown to control the dynamic contact angle $\theta_{d}$ at the fluid front (Fig. 1C) according to the Voinov-Tanner-Cox law, such that $\theta_{d}^{2} \sim C a^{m}$, where $m=1$ for a Newtonian fluid (23), $m>1$ for a viscoelastic fluid (24), and $m<1$ for shear thinning fluids (25). The positive relationship between $\theta_{d}$ and $C a$ shows that the steeper the contact angle, the faster/more viscous the flow (or the lower the cohesion/surface tension). This provides a link between finger morphology and dynamics, and because both wavelength and contact angle depend on $\mathrm{Ca}$, we would expect a power-law trend between the two of the form $\frac{\lambda_{c f}}{h} \sim \theta_{d}^{-1 / m}$.

\section{Solifluction Lobes as Fluid-Like Instabilities}

We argue that the solifluction phenomenon qualitatively exhibits all of the necessary ingredients for a fluid-like instability. Here we describe how each ingredient may translate to soil, resulting in a conceptual model of solifluction pattern formation (Fig. 2C).

Contact line instabilities initiate at a raised fluid front. For solifluction, we propose that a downslope instability forms evenly spaced solifluction terraces that operate similarly to a fluid front. With raised fronts $\sim 1$ to $2 m$ tall and downslope wavelengths $\lambda_{t}$ much larger than soil thickness $\left(\lambda_{t} \sim 10^{1}\right.$ to $\left.10^{2} \mathrm{~m}\right)$ (Figs. $1 A$,
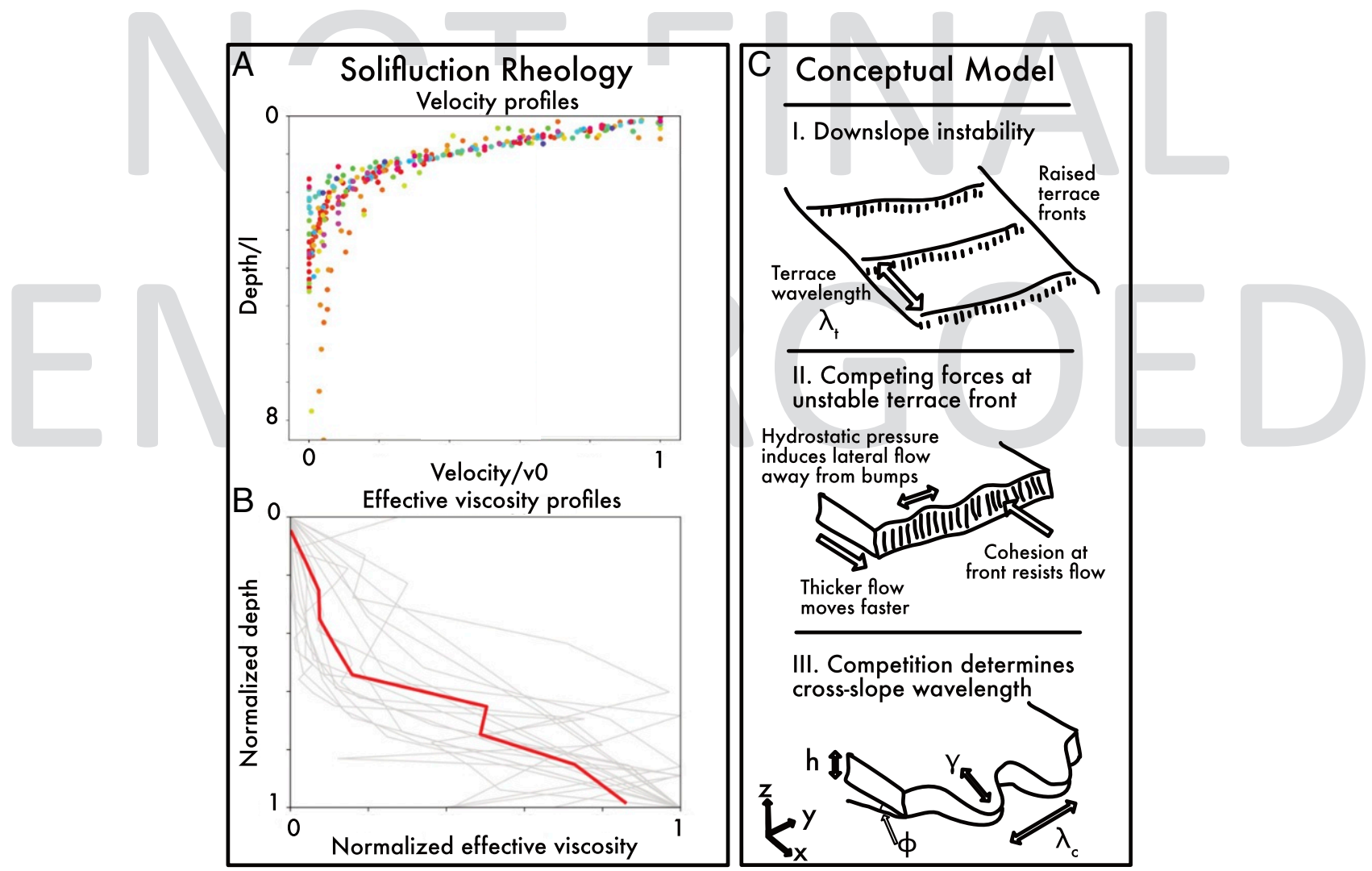

Fig. 2. (A) Vertical velocity profiles compiled from the literature, observed both in the field $(35,38-43)$ and in laboratory experiments (1, 44). Data are normalized by the e-folding depth of exponential fits to each profile (Materials and Methods). See nonnormalized plots in SI Appendix, Fig. S3. ( $B$ ) Vertical viscosity profiles computed from the velocity profiles show a general increase in effective viscosity with depth. The gray lines show individual profiles, while the red line shows the viscosity value averaged all profiles. Data are normalized by max and min values in order to plot between 0 and 1 . See nonnormalized plots in SI Appendix, Fig. S4. (C) Conceptual model of solifluction lobe pattern formation, with variables used in the wavelength scaling analysis defined in Fig. C, III. 
$2 C$, and $3 E$ ), this downslope instability features prominently in the landscape. Although the cause of the downslope instability is unclear, we argue it is likely a result of soil rheology, similar to noninertial waves recently observed in shear thickening fluids or fluids with resisting forces at the free surface (26) (Discussion). With enough heterogeneity in topography, soil properties (such as moisture, cohesion, and grain size), or vegetation, smooth terrace fronts may break into solifluction lobes evenly spaced cross-slope (Figs. $1 A$ and $B$ and $2 C$ ) with cross-slope wavelengths $\lambda_{c}$ on the order of 1 to $10^{2} \mathrm{~m}$. Although the thickness, $h$, of these features is large relevant to fluid thin films, $\sim 1 \mathrm{~m}$, the hillslopewide lateral length scale of motion supports the idea that they may behave like thin films (27).

While solifluction rheology and mechanistic relationships between velocity and depth are still unclear, data and models show that velocity likely increases with total active soil thickness due to freeze-thaw processes $(2,28)$. Field measurements across the globe have found solifluction velocities ranging from $10^{-1}$ to $10^{1} \mathrm{~cm} / \mathrm{y}(2)$. Considering the soil as a slow-moving fluid, these slow velocities suggest very high viscosities. We compile every available field-measured and experimental vertical velocity profile from the literature and find that most exhibit an exponen-

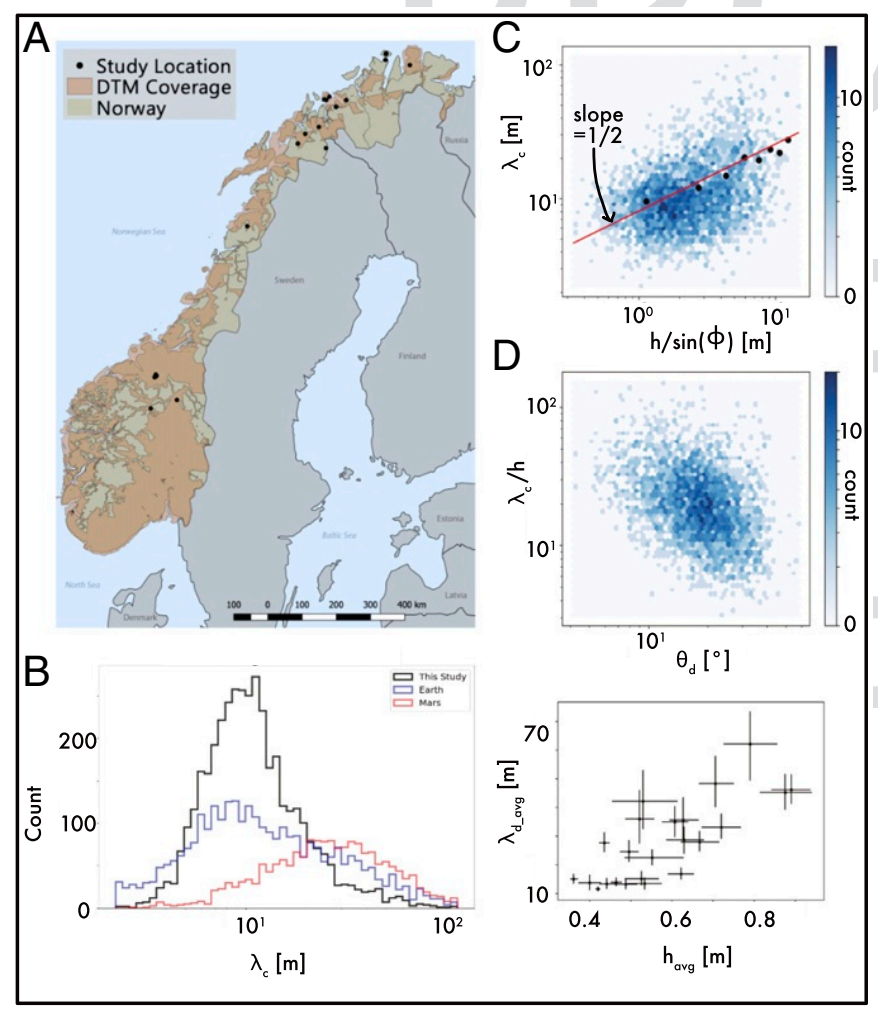

Fig. 3. (A) Map of Norway showing study sites used in this paper and digital terrain map (DTM) coverage. $(B)$ Cross-slope wavelength $\left(\lambda_{c}\right)$ distributions measured in this study, shown with distributions for Earth and Mars from ref. 37. (C) Cross-slope wavelength $\left(\lambda_{c}\right)$ vs. lobe thickness $h /$ topographic slope $\sin \phi$. Due to a large number of data points, data are collected into hexagonal bins with color representing the count in each bin. Darker blue indicates a larger number of data points. The red line shows theoretical prediction from Eq. 7, not a fit to the data; however, vertical position of the line is determined by the best-fit power-law intercept on the raw data. Black dots show average wavelength split into 10 bins of $h / \sin \phi$ values. We omit the highest bin because it contains only one data point and therefore is not a meaningful average. $(D)$ Cross-slope wavelength $\left(\lambda_{c}\right)$ normalized by thickness $h$ vs. contact angle $\theta_{d}$ at the front of the lobe. Data are collected into hexagonal bins with color representing the count in each bin. Darker blue indicates a larger number of data points. $(E)$ Downslope terrace wavelength $\left(\lambda_{t}\right)$ averaged at each site vs. average lobe thickness for each site. tial decrease in velocity with depth (Fig. $2 A$ ) while a few studies exhibit more complex profiles (SI Appendix, Fig S2). We then calculate effective viscosity $\mu_{\text {eff }}$ as the ratio between shear stress $\tau$ and strain rate $d u / d z$, where $u$ is the downslope velocity and $z$ is the vertical depth into the soil profile: $\tau=\mu_{\text {eff }} \frac{d u}{d z}$. We find large $\mu_{\text {eff }}$ ranging from $10^{5}$ to $10^{12} \mathrm{~Pa}$-s. In contrast to a Newtonian fluid with constant viscosity, velocity profiles show that effective viscosity increases with depth (Fig. $2 B$ ), indicating a non-Newtonian-like flow behavior. While a proper description of solifluction rheology should explicitly take into account granular physics, our first-order assumption of non-Newtonian fluid-like behavior is likely acceptable for a wet granular material (29) (Discussion).

Surface tension at the front is the last key ingredient for a contact line instability, as it allows the fluid to thicken and become unstable. Recent studies have shown that intergranular cohesion can produce an effective surface tension in granular materials at small length scales (13); analogous to molecular surface tension, an effective granular surface tension can be calculated as the work required to separate two grains divided by their crosssectional area (13). While this effect may exist in soils, it is likely not physically relevant for $\sim 1-m$-thick solifluction lobes in which overburden pressure vastly outweighs any possible pressure due to effective surface tension. However, there are many sources of cohesion that can lend substantial strength to soils, including microbes (30), permafrost, vegetation (31), capillary bridges due to moisture content (32), clay composition, and solid bridging due to polydispersity (33). Additionally, commonly documented retrograde motion uphill in solifluction lobes points toward strong effects of cohesion in arctic soils $(2,34)$, likely resulting from temporally evolving strength of capillary bridges. We argue that strong soil cohesion and corresponding low soil velocities at solifluction fronts allow soil buildup and transverse flow due to hydrostatic pressure, similar to the behavior of surface tension-dominated fluids. This increased cohesion at the front allows the lobe to maintain its thickness without diffusing away, especially given the steep (often overhanging) slope angle at the front. While to our knowledge no field measurements of soil cohesion trends across a lobe exist, field velocity and morphology measurements support the idea of cohesive, stalled terrace and lobe fronts. Displacement markers in the field show soil buildup behind solifluction lobe fronts and transverse flow toward the middle/front of lobes, similar to behavior in fluid fingers (8). Solifluction terrace and lobe morphology (thickened front and steep, sometimes overhanging contact angle) (8) and dynamics (tractor tread-style rollover motion at the front) $(8,9$, 35 ) resemble those of surface-tension-dominated flows (Fig. 1C). Although the stalling of solifluction fronts is clear from observations, existing field data are not sufficient to determine the physical mechanisms for this observation (however, see Discussion for potential mechanisms). We proceed with our analysis based on the observation of stalled lobe fronts, but without any assumptions of the mechanism for increased cohesion at the front.

\section{Conceptual Model}

We propose that the solifluction lobe instability is initiated and controlled by competition between these elements: 1) the body force due to gravity, which moves thicker material downhill faster; 2) cohesion at the front, which resists flow; and 3) lateral flow due to hydrostatic pressure under topographic bumps (Fig. $2 B$ ), with cross-slope wavelengths set by these competing processes (Fig. 2B). This is similar to fluid contact line fingering in that competition between a body force and resisting force due to cohesion at the front initiates and controls the preferred wavelength of the instability, where increased cohesion at the front takes the place of surface tension. Finally, while formulations of 
fluid contact line instabilities ignore hydrostatic effects because surface tension dominates, here we include hydrostatic pressure that drives lateral flow in the presence of inevitable topographic roughness in natural landscapes.

We develop our analysis to be as general as possible, without assuming a specific source of cohesion at the front of the lobe. While vegetation has been shown to be important for solifluction patterns (36), the existence of nonvegetated lobes precludes vegetation as a necessary ingredient for their formation. Here we focus on solifluction lobes without large boulders; however, stone-banked lobes exhibit grain size segregation with large boulders at the front and sides of the lobe (8). This likely leads to a similar effect in which boulder jamming at the front of the lobe stalls flow. Thus, our general conceptual model should apply to both turf-banked and stone-banked lobes on Earth and Mars, as well as unvegetated lobes with relatively homogeneous grain sizes as are observed on Mars (37).

\section{Wavelength Scaling Analysis}

Inspired by fluid theory for contact line instabilities, we take the first step toward deriving an expression for solifluction lobe wavelengths. Because solifluction rheology is uncertain (but certainly nonlinear, see Fig. 2), our analysis avoids assumptions of Newtonian flow. In contrast to instabilities in surface-tensiondominated fluids, we allow for hydrostatic effects given the likelihood of natural topographic roughness in the field. We examine laminar flow down a plane, accounting for hydrostatic pressure in both the downslope $(x)$ and cross-slope $(y)$ directions. Cohesion has been shown to control effective viscosity in granular materials $(29,45)$. Therefore, to account for cohesion at solifluction fronts, we allow effective viscosity to vary in the $(x)$ direction. Here we present the simplest approach to scaling; see SI Appendix, section I for alternative approaches that produce similar results.

For a laminar fluid flowing down an inclined plane, under hydrostatic conditions upstream from the front, the basal shear stress is

$$
\tau_{0}=-\rho g h \sin \phi+\rho g h \frac{\partial h}{\partial x},
$$

where $\rho$ is the bulk density, $g$ is gravity, $h$ is the fluid thickness, and $\phi$ is the underlying slope. To avoid assumptions of Newtonian rheology, but without assuming a particular form of a power-law fluid, we define a bulk viscosity $\mu$ such that $\tau_{0}=-\mu U / h$, where $U$ is the vertically averaged velocity in the $x$ (downhill) direction. This is akin to using a characteristic viscosity as done in previous non-Newtonian fluid fingering studies in which viscosity varies with depth (19). To account for cohesion at the front, we allow viscosity to change in the $x$ direction. Solving for the downslope velocity and assuming that cross-slope velocity arises only from the hydrostatic pressure gradient, we can solve the continuity equation at steady state and retain only first-order terms (Materials and Methods) to find

$$
\frac{3 \sin \phi}{\mu} \frac{\partial h}{\partial x}-\frac{h \sin \phi}{\mu^{2}} \frac{\partial \mu}{\partial x}-\frac{h}{\mu} \frac{\partial^{2} h}{\partial x^{2}}+\frac{h}{\mu} \frac{\partial^{2} h}{\partial y^{2}}=0,
$$

where the first two terms represent the body force, the third term is the downslope hydrostatic component ( $x$ direction), and the fourth term is the cross-slope hydrostatic component $(y$ direction). Now we can scale terms by dimensionless quantities (indicated with hats) as follows:

$$
\begin{array}{r}
h=h_{0} \hat{h} \\
\mu=\mu_{0} \hat{\mu} \\
x=\gamma \hat{x} \\
y=\lambda \hat{y},
\end{array}
$$

where $h_{0}$ is a characteristic thickness, $\mu_{0}$ is a characteristic viscosity, $\gamma$ is a characteristic length scale in the $x$ direction that describes a distance over which the viscosity varies, and $\lambda$ is a characteristic length scale in the $y$ direction. Retaining only the dimensional leading coefficients and simplifying,

$$
\frac{2 \sin \phi}{\gamma}-\frac{h_{0}}{\gamma^{2}}+\frac{h_{0}}{\lambda^{2}}=0
$$

We note that the viscosity cancels out, and its only effect lies in $\gamma$; thus, the large range of effective viscosity values found in Fig. $2 B$ does not influence the expected scaling proposed here. We are mainly interested in $\lambda$, which we assume to be equivalent to the cross-slope wavelength $\lambda_{c}$ between solifluction lobes (analogous with the wavelength of fluid fingers at a contact line as shown in Eqs. 1 and 2). Assuming the body force (first term) dominates over the downslope hydrostatic pressure gradient (second term), we find

$$
\lambda_{c} \sim \sqrt{\frac{h_{0} \gamma}{2 \sin \phi}} .
$$

This suggests that the cross-slope wavelength increases with soil thickness and the characteristic length over which viscosity changes due to dynamics at the front and decreases with basal slope (which we assume to be equivalent to $x$ directed topographic slope averaged over a distance much greater than length of a lobe). Although the particular scaling differs from that for fluids in Eq. 1, our relationship is similar in that cross-slope wavelength is projected to exhibit a power-law increase with thickness and cohesion and a decrease with topographic slope. These fundamental similarities between solifluction lobe and fluid finger wavelengths also suggest that while we do not yet have a prediction for the contact angle at the front of lobes, we might expect an inverse relationship between cross-slope wavelength normalized by thickness and the contact angle as described above for fluids.

\section{Solifluction Patterns in Norway}

To explore these ideas in real landscapes, we collected highresolution morphologic and topographic data from 26 highly patterned solifluction sites across Norway (Fig. 3). We manually measured 3,000 individual lobes from submeter Light Detection and Ranging (LiDAR)-derived digital elevation models (DEMs) (freely available at Hoydedata) to obtain cross-slope lobe wavelength $\lambda_{c}$, thickness $h$, lobe length $L$, lobe front/riser angle $\theta_{d}$ (hereafter referred to as contact angle), terrace (downslope) wavelength $\lambda_{t}$, and topographic slope angle $\phi$ (Materials and Methods). We find that cross-slope wavelengths range from 2 to $100 \mathrm{~m}$, with a mean of $13 \mathrm{~m}$. This range agrees with previous studies (37), and values are generally smaller but overlap with those found on Mars (Fig. 3B). Trends between lobe morphology metrics and topography agree with theoretical predictions. Crossslope wavelength increases with lobe thickness/topographic slope, as expected from our scaling analysis (Eq. 7). Although the data include a large amount of scatter, binned average wavelengths show that our theoretical prediction describes the general trend well (Fig. 3C). Note that to better explain the data we would need constraints on $\gamma$, which may also depend on lobe thickness and explain the jellybean shape of the data. A better understanding of rheology could also be incorporated in our analysis to improve predictions. Our theory predicts only scaling rather than absolute wavelengths; however, we calculate the best-fit power law coefficient on the raw data $(\approx 8)$ to empirically estimate a coefficient for Eq. 7. Adding a factor of 8 to the front of Eq. 7 and simplifying suggests that cross-slope wavelength $\lambda_{c} \approx 6 \sqrt{h_{0} \gamma / \sin \phi}$.

As expected from theory, we see a negative power-law trend between wavelength/thickness and contact angle. This 
observation is consistent with theory for the dynamic contact angle of a droplet rolling down a flat substrate. However, to properly predict the relationship between cross-slope wavelength and contact angle, we would need theory equivalent to the Voinov-Tanner-Cox law that accounts for cohesion rather than surface tension. We also find that lobe aspect ratio (L/W) slightly increases with topographic slope, as observed in fluid experiments (SI Appendix, Fig. S5) (46). Most lobes are wider than they are long, exhibiting a sawtooth shape similar to that observed for fluids on gently sloping planes (SI Appendix, Fig. S5) (Fig. $1 B$ ). Finally, we observe a positive relationship between downslope terrace wavelength and lobe thickness averaged by site (Fig. $3 E)$, but no clear relationship with topographic slope is discerned (SI Appendix, Fig. S6). While we currently lack a prediction for the scaling of downslope wavelength, our data provide a step toward developing a better understanding of the phenomenon (Discussion).

Large amounts of scatter in the field data likely contain interesting information about lithology, vegetation, climate, and other unknown parameters that differ between sites. However, that average wavelength trends agree with our theory inspired by simple fluids is remarkable and supports the idea that solifluction patterns operate similarly to fluid contact line instabilities.

\section{Climate Controls}

Our data show a meaningful increase in solifluction lobe thickness and cross-slope wavelengths with elevation (Fig. 4), pointing toward a climate control on lobe morphology and pattern formation due to the lapse rate or change in temperature with altitude. Although solifluction features are traditionally thought to be climate controlled and have often been used to interpret past climate, limited data exist for colocated climate metrics and solifluction lobe morphology and dynamics (47). However, recent work on frost cracking in rock (48-50) illuminates the climatic conditions required for segregation ice growth and frost heave, the main drivers of solifluction $(2,51)$. Ref. 49 finds that the depth and intensity of frost cracking increase with annual temperature amplitude and decrease with MAAT. To explore this idea, we compare high temporal resolution climate metrics from extensive monitoring stations in Norway over the last 20 y (52) with solifluction lobe morphology for each site shown in Fig. $3 A$. Consistent with frost cracking predictions, we find

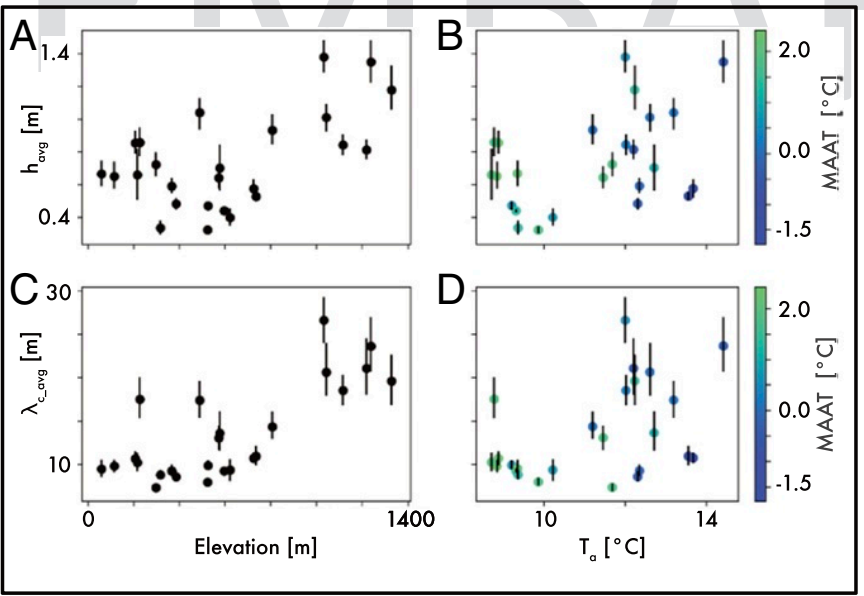

Fig. 4. $(A-D)$ Relationships between lobe morphology, elevation, and climate indexes. Climate data are drawn from daily observations between the years 2000 to $2020 . h_{a v g}$ and $\lambda_{\text {cavg }}$ are the average lobe thickness and crossslope wavelength at each site, respectively. Elevation is given with reference to sea level. $T_{a}$ is the mean annual temperature amplitude, and MAAT is the mean annual air temperature. Vertical bars represent $95 \%$ confidence intervals from the field data averaged at each site. an increase in finger wavelength and lobe thickness with annual temperature amplitude, corresponding with a general decrease in MAAT (Fig. 4). Other differences between high and low elevations may explain observed morphology trends. While we do not see strong relationships with mean annual snowfall, precipitation, or time spent in the frost cracking window (SI Appendix, Figs. 7-9), shortwave radiation or vegetation coverage may be important. We interpret the data to show that climate primarily affects the depth of solifluction processes, which in turn affects the wavelengths. This is supported by a much weaker relationship between elevation and wavelength normalized by thickness (SI Appendix, Fig 10). We acknowledge that we have no constraints on the age or current activity of the features at our sites; therefore, modern climate conditions may not reflect conditions at the time of formation. However, modern studies have found that solifluction processes are active on the Norway mainland (53-55).

\section{Discussion}

Our work suggests that even extremely slow-moving soils may exhibit subcritical fluid-like instabilities, but at length and time scales orders of magnitude larger than those observed in thin films. Our conceptual model for solifluction pattern formation provides a framework for further study. Here we provide some discussion on the most interesting questions resulting from this study, with suggestions for the most promising avenues of exploration.

The initial downslope instability that forms solifluction terraces, which we argue promote the growth of solifluction lobes as a contact line instability, deserves further inspection. While terraces resemble roll waves seen in inertial fluid flows (56), buckling instabilities seen in multilayer flows (57) like rock glaciers (58) and lava flows (59), or wrinkling instabilities found in multilayer solids like pumpkins and human skin (60), our observations of solifluction terrace wavelengths do not fit within these frameworks. Exceedingly slow solifluction velocities exist in a noninertial regime, which precludes a comparison with roll waves (56). While the positive relationship between downslope wavelength and lobe thickness is similar to that seen in buckling and wrinkling instabilities, absolute terrace wavelengths can be much larger than lobe thickness, which is unusual for buckling and wrinkling instabilities; our data show that terrace wavelengths are one to two orders of magnitude larger than lobe thicknesses (Fig. 2E). Further, the observed low effective viscosities at the surface do not align with buckling instabilities, which typically require a more rigid flow on top (58). However, recent work describes a newly observed noninertial instability in shear-thickening flows (e.g., cornstarch mixed with water) that can produce wavelengths much larger than flow thickness (26). These instabilities are shown to result from flow rheology alone and simply require a rheological curve that exhibits shearthickening behavior. Our observations of soil velocity profiles, in which effective viscosity increases with depth and therefore shear stress, may align well with a shear-thickening type rheology. Further, our proposed increase in cohesion at soil fronts may also result in an added free surface stabilizing force, which could allow the instability even without shear-thickening behavior (26). Further study of these "oobleck waves" may inform the critical conditions necessary for solifluction terrace formation; in turn, field studies of solifluction may provide a natural example of similar instabilities at exceedingly low Reynolds number, illuminating our understanding of subcritical fluid instabilities.

While we treat solifluction as a non-Newtonian fluid for a first approach, more study is needed to understand the complex rheology of soliflucting soil from a granular perspective. Granular flow rheology is currently understood within the $\mu(I)$ framework, a dimensionless form of the classic shear stress/strain 
rate relationship that accounts for confining pressure relevant for granular materials $(61,62)$. In essence, $\mu(I)$ is very similar to fluid rheology, but allows for the role of changing confining pressure with depth. However, the extremely low solifluction velocities observed in the field indicate that solifluction occurs not as a granular flow but well within the granular creep regime (63) that has been shown to describe soil transport velocities on temperate hillslopes $(64,65)$. Granular creep rheology is still at the forefront of granular physics research. Experiments have shown that creep occurs below the assumed static coefficient of friction (63). While creep rheology is still uncertain, new models for creep indicate that rather than a viscous-like flow rule, an elastoplastic model may be physically relevant (66). Interestingly, a similar type of model was found to best describe solifluction experiments, rather than a viscous model (67). Experimental work and field work are needed to understand whether solifluction is best described as a creeping granular material, a highly viscous non-Newtonian fluid, or some combination of the two, especially given the complex, temporally changing processes (frost heave, gelifluction) that are known to drive it.

Our results also suggest strong connections between climate and solifluction lobe morphology. While much more detailed work is needed to quantitatively understand the role of climate in setting solifluction patterns and lobe morphology, these results suggest that lobe morphology metrics measurable from remotesensing data may contain information about present and past climate, both on Earth and on other planets. Additionally, these data show that a changing climate may have substantial effects on solifluction dynamics and morphologies. This relates to a fundamental, yet unanswered question: Why do we see solifluction patterns only in cold places? We argue that solifluction provides an example of a contact line instability in a parameter space well outside that of previous studies, with the potential to help shed light on recently observed subcritical fluid instabilities $(26,46)$ and unstable behavior of soft materials (62). Strong heterogeneity in topography and material properties may be required for the instability to form, as is observed in subcritical fluid fingering over rough substrates $(46,68)$; it is notable that many hillslopes exhibit solifluction terraces with smooth fronts that are not broken into fingers; ref. 8 qualitatively noted that terraces seem to form in areas with homogeneous snow cover and smooth topography, further supporting the idea that solifluction lobes grow as a secondary instability on top of the downslope instability and require heterogeneity to form. We do acknowledge that isolated solifluction lobes are also observed in areas with increased soil moisture (8), perhaps behaving similarly to an isolated droplet moving down a plane (69). Numerical and appropriately scaled physical experiments may be used to explore required thresholds for the onset of both the downslope and cross-slope instabilities under different rheological regimes and to test the idea that competition between gravity and cohesion is needed to initiate the instability. A better understanding of critical conditions for the onset of the instabilities would also inform our understanding of solifluction lobes seen on Mars, whether they require a cold climate to form, and what explains the larger wavelengths seen on Mars $(6,37)$. Our findings may also have relevance for earthflows, temperate, slow-moving landslides that exhibit similar morphologic and dynamic characteristics to solifluction lobes $(70)$.

More work is needed to understand soil cohesion and velocity trends across solifluction features and how they relate to the onset of instability. We highlight three potential mechanisms for observed spatial gradients in soil velocities, all related to soil moisture: capillary suction, ice lens formation, and vegetation. Studies of soil moisture trends across lobes are limited and find conflicting results that may point toward different mechanisms for increased cohesion at the front; refs. 8 and 71 find that lobes are drier at the front due to drainage, while refs. 36 and 72 find increased soil moisture at lobe fronts due to lower permeability and funneling of water along lobes. Either case may lead to increased cohesion at the front. In the dry front case, decreased soil moisture may induce high capillary suction and therefore higher cohesion (73-75). Lower soil moisture may also inhibit the formation of ice lenses, which drive frost heave and need moisture to grow $(76,77)$. The author of ref. 8 found that the water table was lowest at the boundaries of solifluction lobes during the fall freeze-up, which he interpreted limited the formation of ice lenses and explained low soil velocities at the front. In contrast, increased soil moisture may promote preferential growth of vegetation at the front (36) that adds cohesion through root strength. It is also noteworthy that soil moistures recorded by ref. 72 fall in the $\sim 5$ to $10 \%$ range, corresponding to strong capillary suction (73-75). Finally, compaction of the front may reduce porosity and permeability $(72,78)$, altering the rheology of the soil and decreasing the efficiency of ice lensing if permeability is low enough (77). It is possible that all of these effects occur and are important at different points in the season; for example, increased soil moisture at the front may promote vegetation growth in the spring, and decreased moisture after drainage decreases ice lens formation in the fall. Targeted field studies are needed to uncover spatial and temporal changes in soil cohesion across lobes and its effects on stalling the lobe front.

Our analysis is targeted at behavior at the onset of the solifluction lobe instability. Once initiated, the pattern will be selfenhanced as the increased resistance at the raised lobe fronts will further stagger the flow. Nevertheless, more work is needed to understand the evolution of these features through time, as well as possible merging of lobes that would skew measurements toward larger wavelengths. Field studies could examine how disparate lobes interact; for example, once formed, the presence of lobes can redirect water flow through the landscape, influencing lobe development and initiation upslope/downslope (72). For the downslope instability, studies that examine downslope patterns in terrace front exposure dates could determine whether these waves form all at once or initiate at the bottom of a slope and propagate upward. The presence of lobes may also exert a weathering feedback on the underlying bedrock and permafrost, as soil thickness changes substantially along the length of a lobe.

Finally, our results highlight the importance of cohesion in landscape evolution. Rather than simply increasing shear strength, as typically assumed in Mohr-Coulomb soil mechanics models, we suggest that the presence of cohesion can lead to nonlinear dynamics that cause large-scale instabilities in landscapes. While further field and experimental work is needed to better understand the rheology of arctic soils, we suggest that incorporating formulations of cohesion into soil transport models is key to accurately predict landscape evolution and response to climate change.

\section{Materials and Methods}

Velocity and Viscosity Profiles. We used WebPlotDigitizer (https://automeris io/WebPlotDigitizer/) to digitize solifluction lobe velocity profile data from the literature, both from field $(35,38-43)$ and from laboratory experiments $(1,44)$. To make the plots shown in ref. 2 , we compile the data in python and ensure all data are in the same units. For velocity profiles we attempt to fit an exponential line to the data; if the fit is arbitrarily better than 0.85 (most profiles), then we calculate an e-folding depth to collapse the data onto a normalized plot. If the fit is worse than 0.85 , we present those profiles nonnormalized in SI Appendix. To calculate effective viscosity, we calculate the change in velocity with depth between each data point to obtain the strain rate. We calculate shear stress as $\tau=\rho g z s$, where we use a constant bulk density $\rho$ of $2,500 \mathrm{~kg} / \mathrm{m}^{3}, g=9.8, z=$ depth in meters, and slope values given in each individual study from which the data are obtained. We then calculate effective viscosity as the ratio between shear stress and strain rate. 
Finally, we average over all profiles by $10-\mathrm{cm}$-wide depth bins to calculate an average effective viscosity profile for all of the data.

\section{Lobe Wavelength Data.}

Wavelength calculations. Study sites were selected using a combination of high-resolution orthophotos and a hillshade of the digital elevation model. We selected 30 hillslopes on the order of 500 to $1,000 \mathrm{~m}$ long where solifluction was the dominant topographic pattern throughout the domain. Sites with exposed bedrock, gullies, or ponds were avoided. Using a gradient and hillshade map, cross-hillslope groups of solifluction lobes were manually delineated (SI Appendix, Fig. S1). To streamline and standardize the delineation process, we represent each lobe as a georeferenced triangle. The three vertices defining the triangle were placed along the riser of the lobe at the apex and the two points on either side of the apex where adjacent lobes begin (SI Appendix, Fig. S1). Lobes were not delineated when riser edges and transitions into adjacent lobes were ambiguous. In addition, some sites contained smaller lobes superimposed on larger terraces or lobes. In these instances we delineated the smaller-scale feature. In addition to individual lobes, a minimum of five downslope transects were delineated at each study site. Transects were oriented in the direction of the lobes with vertices added each time the transect crossed the riser of a lobe. Over 3,500 individual lobes were delineated across 28 hillslopes.

For each lobe we used the triangle vector to estimate several planform morphological metrics including lobe orientation, width, and length. To determine orientation we first calculated the line bisecting the interior angle at the apex of the lobe. Lobe orientation was taken to be the direction of this line. Lobe width was calculated as the distance between the two endpoints on either side of the apex. Lobe length was calculated as the minimum distance between the apex and the line connecting the two endpoints. At each lobe a local transect was extracted from the elevation data using a 50-m window centered at the lobe apex and in the direction of the bisecting line. Elevation profiles along the transect were extracted using linear interpolation with the number of points in the profile determined by the length of the transect and the DEM resolution (length/cell size) (SI Appendix, Fig. S1 B and C).

From the profile, lobe thickness and contact angle (referred to as riser angle in the solifluction literature) were determined. Transects were first detrended by finding the best-fit line to the entire $50-\mathrm{m}$ transect in a leastsquares sense. The slope of the trend line was taken to be the parent slope. To calculate lobe thickness and contact angle, the detrended profile is subset to include only the portion of the profile representing the manually delineated lobe and $2 \mathrm{~m}$ downslope of the lobe apex (SI Appendix, Fig S1D). Thickness is calculated as the elevation range in the subsetted profile. Contact angle is calculated as the maximum derivative along the subsetted profile using a central differencing scheme.

To compare data in Fig. 2 with our theoretical prediction, we plot a $1 / 2$ power law on top of the data and use the best-fit power-law intercept to position it. Data are split into $10 x$-axis bins and averaged to give the black data points in Fig. 2.

1. C. Harris, M. C. R. Davies, J.-P. Coutard, Rates and processes of periglacial solifluction An experimental approach. Earth Surf. Process. Landforms J. Brit. Geomorph. Group 22, 849-868 (1997).

2. N. Matsuoka, Solifluction rates, processes and landforms: A global review. Earth Sci. Rev. 55, 107-134 (2001)

3. C. Harris et al., Solifluction processes on permafrost and non-permafrost slopes: Results of a large-scale laboratory simulation. Permafr. Periglac. Process. 19, 359-378 (2008).

4. J. C. Rowland et al., Arctic landscapes in transition: Responses to thawing permafrost Eos Trans. Am. Geophys. Union 91, 229-230 (2010).

5. L. Kondic, J. Diez, Pattern formation in the flow of thin films down an incline: Constant flux configuration. Phys. Fluids 13, 3168-3184 (2001).

6. A. Johnsson et al., Periglacial mass-wasting landforms on Mars suggestive of transient liquid water in the recent past: Insights from solifluction lobes on Svalbard. Icarus 218, 489-505 (2012).

7. H. E. Huppert, Flow and instability of a viscous current down a slope. Nature $\mathbf{3 0 0}$, 427-429 (1982)

8. J. B. Benedict, Downslope soil movement in a Colorado alpine region: Rates, processes, and climatic significance. Arct. Alp. Res. 2, 165-226 (1970)

9. G. Elliott, Microfabric evidence for podzolic soil inversion by solifluction processes. Earth Surf. Process. Landforms 21, 467-476 (1996).

10. I. Veretennikov, A. Indeikina, H.-C. Chang, Front dynamics and fingering of a driven contact line. J. Fluid Mech. 373, 81-110 (1998).

11. S. M. Troian, E. Herbolzheimer, S. A. Safran, J. F. Joanny, Fingering instabilities of driven spreading films. Europhys. Lett. 10, 25 (1989).

12. R. W. Style, A. Jagota, C.-Y. Hui, E. R. Dufresne, Elastocapillarity: Surface tension and the mechanics of soft solids. Annu. Rev. Condens. Matter Phys. 8, 99-118 (2017).
Climate Data. We use SeNorge2, a gridded meteorological dataset with a spatial resolution of $1 \mathrm{~km}^{2}$ and a temporal resolution of $1 \mathrm{~h}$ to estimate typical climate conditions for each study site. Data are from the Norway Meteorological Organization and can be found at https://thredds.met.no/ thredds/catalog/senorge/catalog.html. While hourly data are available, in this study we used products released at the daily time scale. The variables include maximum daily temperature, minimum daily temperature, mean daily temperature, and daily precipitation. The gridded data are interpolated from monitoring stations throughout Norway and are corrected to account for elevation. For full description of the climate data see ref. 52. We identified each grid cell containing a study site and extracted the previous 20 y of daily climate data. We grouped the mean daily temperature data for each site by year and then calculated the yearly temperature amplitude as the difference between max and min mean daily temperature for each year. We then report mean yearly temperature amplitude $T_{a}$ as the mean temperature amplitude averaged over all $20 \mathrm{y}$ of data for each site bounded within the 5th and 95th percentiles of the data. We calculated the number of frost cycles per year at each site where a frost cycle was defined as a zero crossing of the temperature data. Since the hourly data are summarized at the daily scale, this is equivalent to a change in sign between the maximum daily temperature and the minimum daily temperature. We used the surface temperature data as a proxy for ground temperature (i.e., no corrections/adjustments are made). Justification comes from experimental studies measuring soil movement due to frost heave and gelifluction. We averaged the morphology data at each site to compare with the number of frost cycles and bootstrapped $95 \%$ confidence intervals for the means.

Data Availability. All data and code used to produce the figures are available at the Next Generation Ecosystem Experiments (NGEE) Arctic Data Repository (79) (https://doi.org/10.5440/1768024). Norwegian LiDAR data are available for download at https://hoydedata.no/LaserInnsyn/. Norwegian climate data are available at https://thredds.met.no/thredds/catalog/ senorge/catalog.html. Additional figures and text are provided in $\mathrm{SI}$ Appendix.

ACKNOWLEDGMENTS. We thank David Furbish for substantial help in developing the wavelength scaling analysis; Bob Anderson for getting us on the solifluction pattern train in the first place; Doug Jerolmack for informative discussions about granular rheology; Mara Nutt for help with data collection during Lobe-a-thon 2020; two anonymous reviewers whose comments greatly improved the manuscript; and Nakul Deshpande, Andreas Johnsson, Michael Davies, Joanmarie Del Vecchio, Charlie Shobe, Anastasia Piliouras, Jon Schwenk, Yu Zhang, Sajjan Heerah, Cyrano de Bergerac, and Will McDermid for valuable discussions and proofreading. This research was conducted under the Next Generation Ecosystem Experiments Arctic (NGEE-Arctic) project (Department of Energy [DOE] ERKP757), funded by the Office of Biological and Environmental Research in the US DOE Office of Science.

13. J. R. Royer et al., High-speed tracking of rupture and clustering in freely falling granular streams. Nature 459, 1110-1113 (2009).

14. R. Brewster, G. S. Grest, A. J. Levine, Effects of cohesion on the surface angle and velocity profiles of granular material in a rotating drum. Phys. Rev. E 79, 011305 (2009).

15. G. Prado, Y. Amarouchene, H. Kellay, Experimental evidence of a Rayleigh-Plateau instability in free falling granular jets. Phys. Rev. Lett. 106, 198001 (2011).

16. L.-H. Luu, G. Castillo, N. Mujica, R. Soto, Capillarylike fluctuations of a solidliquid interface in a noncohesive granular system. Phys. Rev. E 87, 040202 (2013).

17. S. R. Waitukaitis, H. F. Grütjen, J.R. Royer, H. M. Jaeger, Droplet and cluster formation in freely falling granular streams. Phys. Rev. E 83, 051302 (2011).

18. N. Mujica, R. Soto, "Dynamics of noncohesive confined granular media" in Recent Advances in Fluid Dynamics with Environmental Applications, G. R. Chavarría, J. Klapp, A. Medina, A. López, L. D. G. Sigalotti, Eds (Springer, 2016), pp. 445-463.

19. J. R. de Bruyn, P. Habdas, S. Kim, Fingering instability of a sheet of yield-stress fluid. Phys. Rev. E 66, 031504 (2002).

20. M. F. G. Johnson, R. A. Schluter, M. J. Miksis, S. G. Bankoff, Experimental study of rivulet formation on an inclined plate by fluorescent imaging. J. Fluid Mech. 394, 339-354 (1999).

21. M. A. Spaid, G. M. Homsy, Stability of Newtonian and viscoelastic dynamic contact lines. Phys. Fluid. 8, 460-478 (1996).

22. B. Hu, S. L. Kieweg, Contact line instability of gravity-driven flow of power-law fluids. J. Non Newtonian Fluid Mech. 225, 62-69 (2015).

23. O. V. Voinov, Hydrodynamics of wetting. Fluid Dynam. 11, 714-721 (1976)

24. J.-H. Kim, H. P. Kavehpour, J. P. Rothstein, Dynamic contact angle measurements on superhydrophobic surfaces. Phys. Fluids 27, 032107 (2015). 
25. G. K. Seevaratnam, Y. Suo, E. Ramé, L. M. Walker, S. Garoff, Dynamic wetting of shear thinning fluids. Phys. Fluids 19, 012103 (2007).

26. B. D. Texier, H. Lhuissier, Y. Forterre, B. Metzger, Surface-wave instability without inertia in shear-thickening suspensions. Commun. Phys. 3, 1-7 (2020).

27. R. V. Craster, O. K. Matar, Dynamics and stability of thin liquid films. Rev. Mod. Phys. 81, 1131 (2009).

28. R. S. Anderson, S. P. Anderson, Geomorphology: The Mechanics and Chemistry of Landscapes (Cambridge University Press, 2010).

29. S. Roy, S. Luding, T. Weinhart, A general (ized) local rheology for wet granular materials. New J. Phys. 19, 043014 (2017).

30. S. E. Yasodian, R. K. Dutta, L. Mathew, T. M. Anima, S. B. Seena, Effect of microorganism on engineering properties of cohesive soils. Geomech. Eng. 4, 135-150 (2012).

31. C.-B. Zhang, L.-H. Chen, Y.-P. Liu, X.-D. Ji, X.-P. Liu, Triaxial compression test of soilroot composites to evaluate influence of roots on soil shear strength. Ecol. Eng. 36, 19-26 (2010).

32. M. Pouragha, R. Wan, Non-dissipative structural evolutions in granular materials within the small strain range. Int. J. Solid Struct. 110, 94-105 (2017).

33. A. Seiphoori, X.-G. Ma, P. E. Arratia, D. J. Jerolmack, Formation of stable aggregates by fluid-assembled solid bridges. Proc. Natl. Acad. Sci. U.S.A. 117, 3375-3381 (2020).

34. A. G. Lewkowicz, S. Clarke, "Late-summer solifluction and active layer depths, Fosheim Peninsula, Ellesmere Island, Canada" in Proceedings of the 6th International Conference on Permafrost, A. G. Lewkowicz, M. Allard, Eds. (Centre d'Études Nordiques, Université Laval, Yellowknife, Canada, 1998), pp. 641-666.

35. N. Matsuoka et al., Solifluction resulting from one-sided and two-sided freezing: Field data from Svalbard. Polar Geosci. 13, 187-201 (2000).

36. J. Eichel et al., Solifluction meets vegetation: The role of biogeomorphic feedbacks for turf-banked solifluction lobe development. Earth Surf. Process. Landforms 42, 16231635 (2017).

37. R. Gastineau et al., Small-scale lobate hillslope features on Mars: A comparative 3D morphological study with terrestrial solifluction lobes and zebra stripe lobes. Icarus 342, 113606 (2020).

38. C. K. Ballantyne, A 35-year record of solifluction in a maritime periglacial environment. Permafr. Periglac. Process. 24, 56-66 (2013).

39. D. J. Smith, Rates and controls of soil movement on a solifluction slope in the Mount Rae area, Canadian Rocky Mountains. Zeitschrift für Geomorphologie NF 71, 25-44 (1988).

40. L. W. Price, Subsurface movement on solifluction slopes in the Ruby Range, Yukon Territory, Canada-a 20-year study. Arct. Alp. Res. 23, 200-205 (1991).

41. N. Matsuoka, A. Ikeda, T. Date, Morphometric analysis of solifluction lobes and rock glaciers in the Swiss Alps. Permafr. Periglac. Process. 16, 99-113 (2005).

42. N. Matsuoka, Solifluction and mudflow on a limestone periglacial slope in the Swiss Alps: 14 years of monitoring. Permafr. Periglac. Process. 21, 219-240 (2010)

43. C. Kinnard, A. G. Lewkowicz, Movement, moisture and thermal conditions at a turfbanked solifluction lobe, Kluane Range, Yukon Territory, Canada. Permafr. Periglac Process. 16, 261-275 (2005).

44. C. Harris, "The role of climatic and soil properties in periglacial solifluction: Evidence from laboratory simulation experiments" in Solifluction and Climatic Variations in the Holocene, Special Issue: ESF Project European Paleoclimate and Man , J. A. Matthews, B. Glaser, Eds. (G. Fischer, Stuttgart and New York 1993), vol. 6.

45. M. Macaulay, P. Rognon, Viscosity of cohesive granular flows. Soft Matter 17, 165-173 (2020)

46. J.S. Marshall, R. Ettema, Contact-line instabilities of driven liquid films. WIT Transactions on State-of-the-Art in Science and Engineering, 6 (2005).

47. N. Matsuoka, Climate and material controls on periglacial soil processes: Toward improving periglacial climate indicators. Quat. Res. 75, 356-365 (2011).

48. R. S. Anderson, Near-surface thermal profiles in alpine bedrock: Implications for the frost weathering of rock. Arct. Alp. Res. 30, 362-372 (1998).

49. T. C. Hales, J. J. Roering, Climatic controls on frost cracking and implications for the evolution of bedrock landscapes. J. Geophys. Res. Earth Surface, 10.1029/2006JF000616 (2007).

50. J. A. Marshall et al., Frost for the trees: Did climate increase erosion in unglaciated landscapes during the Late Pleistocene? Sci. Adv. 1, e1500715 (2015).
51. C. Harris, M. C. R. Davies, Gelifluction: Observations from large-scale laboratory simulations. Arctic Antarct. Alpine Res. 32, 202-207 (2000).

52. C. Lussana et al., Senorge2 daily precipitation, an observational gridded dataset over Norway from 1957 to the present day. Earth Syst. Sci. Data 10, 235 (2018).

53. H. Ø. Eriksen et al., Visualizing and interpreting surface displacement patterns on unstable slopes using multi-geometry satellite SAR interferometry (2D InSAR). Remote Sens. Environ. 191, 297-312 (2017).

54. C. Harris, M. Kern-Luetschg, F. Smith, K. Isaksen, Solifluction processes in an area of seasonal ground freezing, Dovrefjell, Norway. Permafr. Periglac. Process. 19, 31-47 (2008)

55. P. Worsley, C. Harris, Evidence for neoglacial solifluction at Okstindan, north Norway. Arctic 27, 128-144 (1974)

56. N. J. Balmforth, S. Mandre, Dynamics of roll waves. J. Fluid Mech. 514, 1-33 (2004).

57. A. C. Slim, J. Teichman, L. Mahadevan, Buckling of a thin-layer Couette flow. J. Fluid Mech. 694, 5-28 (2012).

58. D. S. Loewenherz, C. J. Lawrence, R. L. Weaver, On the development of transverse ridges on rock glaciers. J. Glaciol. 35, 383-391 (1989).

59. R. W. Griffiths, The dynamics of lava flows. Annu. Rev. Fluid Mech. 32, 477-518 (2000)

60. Q. Wang, X. Zhao, A three-dimensional phase diagram of growth-induced surface instabilities. Sci. Rep. 5, 8887 (2015).

61. P. Jop, Y. Forterre, O. Pouliquen, A constitutive law for dense granular flows. Nature 441, 727-730 (2006).

62. D. J. Jerolmack, K. E. Daniels. Viewing Earth's surface as a soft-matter landscape. Nat Rev. Phys. 1, 716-730 (2019).

63. M. Houssais, C. P. Ortiz, D. J. Durian, D. J. Jerolmack, Onset of sediment transport is a continuous transition driven by fluid shear and granular creep. Nat. Commun. 6, 6527 (2015).

64. B. Ferdowsi, C. P. Ortiz, D. J. Jerolmack, Glassy dynamics of landscape evolution. Proc. Natl. Acad. Sci. U.S.A. 115, 4827-4832 (2018).

65. N. Deshpande, D. Furbish, P. Arratia, D. Jerolmack, The perpetual fragility of creeping hillslopes. https://doi.org/10.31223/osf.io/qc9jh (18 May 2020).

66. A. Nicolas, E. E. Ferrero, K. Martens, J.-L. Barrat, Deformation and flow of amorphous solids: Insights from elastoplastic models. Rev. Mod. Phys. 90, 045006 (2018).

67. C. Harris, M. C. R. Davies, B. R. Rea, Gelifluction: Viscous flow or plastic creep? Earth Surf. Process. Landforms J. Brit. Geomorph. Res. Group 28, 1289-130 (2003).

68. J. S. Marshall, S. Wang, Contact-line fingering and rivulet formation in the presence of surface contamination. Comput. Fluid. 34, 664-683 (2005).

69. L. Mahadevan, Y. Pomeau, Rolling droplets. Phys. Fluid. 11, 2449-2453 (1999).

70. P. Lacroix, A. L. Handwerger, G. Bièvre. Life and death of slow-moving landslides. Nat. Rev. Earth Environ. 1, 404-419 (2020)

71. C. Harris, Engineering properties, groundwater conditions, and the nature of soil movement on a solifluction slope in north Norway. Q. J. Eng. Geol. Hydrogeol. 10, 27-43 (1977).

72. D. Draebing, J. Eichel, Spatial controls of turf-banked solifluction lobes and their role for paraglacial adjustment in glacier forelands. Permafr. Periglac. Process. 28, 446-459 (2017)

73. Y. Matsushi, Y. Matsukura, Cohesion of unsaturated residual soils as a function of volumetric water content. Bull. Eng. Geol. Environ. 65, 449-455 (2006).

74. N. Lu, W. J. Likos, Suction stress characteristic curve for unsaturated soil. J. Geotech Geoenviron. Eng. 132, 131-142 (2006).

75. R. Wan, S. Khosravani, M. Pouragha, Micromechanical analysis of force transport in wet granular soils. Vadose Zone J. 13, 1-12 (2014).

76. A. W. Rempel, Formation of ice lenses and frost heave. J. Geophys. Res. Earth Surface, 10.1029/2006JF000525 (2007).

77. A. W. Rempel, J. A. Marshall, J. J. Roering, Modeling relative frost weathering rates at geomorphic scales. Earth Planet Sci. Lett. 453, 87-95 (2016)

78. J. B. Benedict, Frost creep and gelifluction features: A review. Quat. Res. 6, 55-76 (1976)

79. M. Fratkin et al., Arctic soil patterns analogous to fluid instabilities: Supporting data. Next Generation Ecosystem Experiments Arctic data collection(Oak Ridge National Laboratory, US Department of Energy, Oak Ridge, TN) https://doi.org/10.5440/1768024. 